\title{
Is it all just a pipe dream?
}

\author{
Researchers trying to turn nanotubes into storage systems for hydrogen \\ fuel are finding that corporate funding and academic openness can be \\ hard to combine. Catherine Zandonella delves into a carbon controversy.
}

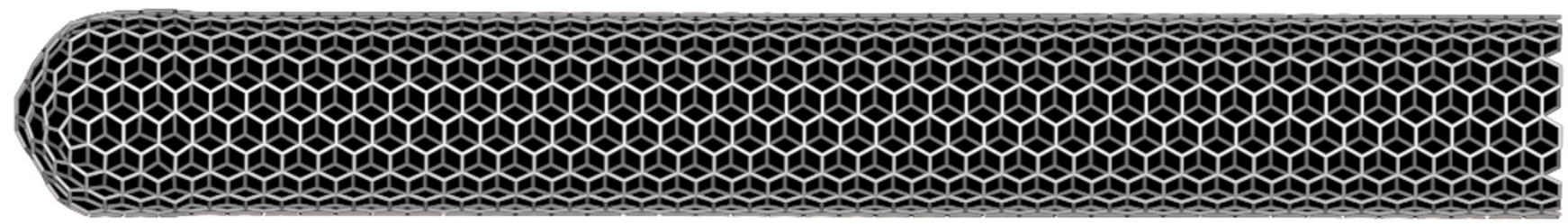

$\mathrm{T}$ he fuzzy black soot at the bottom of the flasks in Michael Heben's lab looks ordinary enough. But for those funding his work at the National Renewable Energy Laboratory in Golden, Colorado, it might someday be worth a fortune. If Heben is right, this grimy substance might be able to store fuel for a new generation of hydrogen-powered cars. But with money and patents at stake, some researchers feel that academic openness is playing second fiddle to corporate interests.

The soot contains carbon nanotubes, tiny hollow cylinders around 1.5 nanometres in diameter. Their walls are made up of a single layer of carbon atoms, and can be thought of as a sheet of atoms arranged in a hexagonal pattern and then rolled up to form a tube much like a miniature roll of chicken wire (see above). Based on the nanotubes first synthesized in 1991 by Sumio Iijima at the NEC Corporation in Japan ${ }^{1}$, these ultrastrong, lightweight tubes have potential uses ranging from wiring in integrated circuits to components in nanoscale motors. And for researchers in the clean-energy business, nanotubes carry the tantalizing potential of being a new storage system for hydrogen fuel.

Billed as the energy source of the future, hydrogen is plentiful and clean. Fuel cells batteries that combine oxygen and hydrogen to produce electricity - could one day power virtually all cars and buses, pumping out nothing more harmful than warm water. But for hydrogen to be a practical fuel for cars of the future, researchers must first develop a lighter storage system to replace the bulky tanks of liquefied or condensed hydrogen found in many of today's electric vehicles.

Carbon nanotubes have the right qualifications for the job. They can be arranged in groups to form rope-like bundles. The diameter of the individual tubes is typically two or three times that of a hydrogen atom, and researchers believe that hydrogen could be tucked both inside and between the tubes. The amount of hydrogen that nanotubes can adsorb in this way depends on pressure and temperature, so changing these conditions should allow hydrogen to be pumped in and out of the system.

\section{Test tubes}

Despite this exciting potential, years of work on nanotubes have produced mixed results. Progress has been made, but funding arrangements are muddying the waters. Some researchers using corporate money are prohibited from fully disclosing their methods until patents are filed, generating doubts about their findings. The simmering tensions came to a head last November at a Materials Research Society (MRS) meeting in Boston. Acrimonious debate erupted after some researchers publicly criticized new results.

The history of hydrogen storage in carbon materials is littered with promising findings that seemed to collapse under closer scrutiny. One frequently cited example is the work of Nelly Rodriguez, a researcher at Northeast-

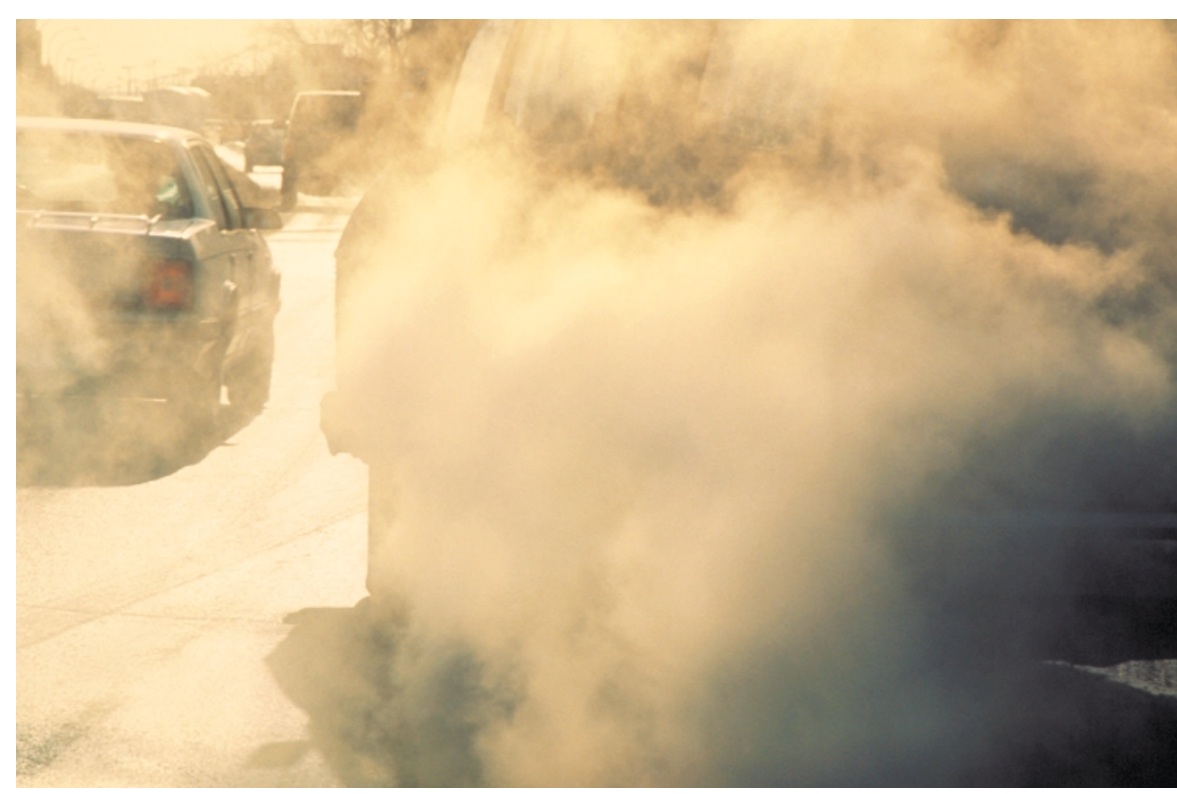

Up in smoke: pollution from today's cars could be eliminated if hydrogen fuel can be stored effectively. ern University near Boston. In 1998, Rodriguez announced that she could get graphite nanofibres - thin wires of carbon - to adsorb their own weight in hydrogen ${ }^{2}$. Citing patent concerns, she refused to reveal the details of her technique, generating widespread scepticism about her results.

So far, no one has managed to reproduce Rodriquez's results ${ }^{3}$. She, in turn, has stopped presenting her findings at meetings because of the criticism she received. Rodriquez is now leaving Northeastern to concentrate on her company, Catalytic Materials, based in Mansfield, Massachusetts, which has the backing of several unnamed corporations. "The project is going extremely well," she says, "but we cannot publish our work because we have contracts that are proprietary. This is one of the reasons we are leaving academia."

Another example is work conducted by Jianyi Lin at the National University of Singapore. In a 1999 paper, Lin and colleagues reported high storage levels in lithium-doped 
multiwalled nanotubes ${ }^{4}$ - tubes that are nested one inside the other like Russian dolls. Lin claimed that $20 \%$ of the combined weight of nanotubes and adsorbed hydrogen was attributable to the hydrogen - described as $20 \%$ by weight. Since then, other researchers have shown that Lin was actually measuring storage of water rather than hydrogen ${ }^{5}$.

But despite these incidents, new results have continued to attract the interest of energy and car companies. A more practical hydrogen storage system would bring huge financial rewards, so corporations are willing to forgive a few false alarms.

\section{Carbon conundrums}

To foster research in this area, the US Department of Energy (DoE) has set target storage levels for vehicle fuel systems. It says that for a practical system, a tank should hold $62 \mathrm{~kg}$ of hydrogen per cubic metre, or $6.5 \%$ by weight. The system needs to adsorb and release hydrogen quickly while working at temperatures ranging from 25 to $100^{\circ} \mathrm{C}$, and preferably at pressures of no more than 200 times normal atmospheric pressure.

Since the guidelines were issued, Heben's team has used a new technique to produce purer nanotubes ${ }^{6}$, eliminating virtually all the carbon not in the form of tubes from the samples. At the International Winterschool on Electronic Properties of Novel Materials, held in March last year in Kirchberg, Austria, he presented spectacular results, beating the DoE goals with storage of up to $7 \%$ hydrogen by weight at room temperature and ambient pressure in tubes he says are up to $98 \%$ pure.

But at November's MRS meeting, Michael Hirscher of the Max Planck Institute for Metals Research in Stuttgart announced that he had repeated Heben's experiments using similar nanotubes and obtained very different results. Not only did Hirscher dispute Heben's figure of $7 \%$ storage - his nanotubes could only store $1.5 \%$ hydrogen by weight - but he found traces of titanium contamination within the tubes. Titanium adsorbs hydrogen, and Hirscher claimed that the metal was responsible for all of the adsorption. The titanium came from a probe used to open the ends of the tubes in an effort to increase their storage capacity. When Hirscher used a stainless-steel probe, the tubes' storage capacity plummeted. Hirscher backed up his claims this February, when he published an invited paper ${ }^{7}$ in the journal Applied Physics A.

Heben was astounded at what he saw as a betrayal of a working relationship with Hirscher. The two had been in contact for some time, and Heben had supplied Hirscher with several key details of the experiment. Heben says that he is well aware of the titanium. Rather than being a contaminant, Heben sees it as vital to the higher storage density but not in the way that Hirscher suggests. Heben agrees that the titanium may adsorb small quantities of hydrogen. But more

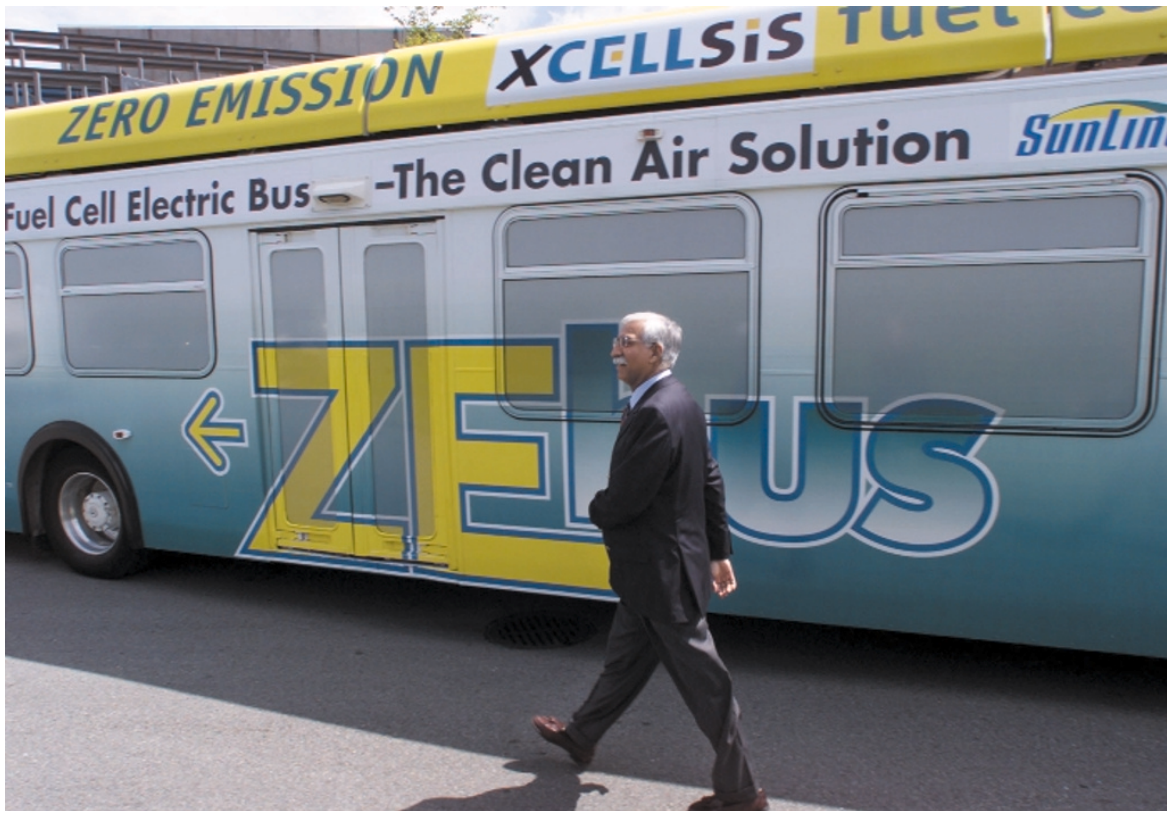

Tanked up: electric buses promote clean air, but require bulky containers to store hydrogen fuel.

importantly, he believes that its presence somehow stimulates the carbon nanotubes to take up more hydrogen — although he admits that he cannot prove this yet. Heben now has a patent for using titanium in carbon nanotubes. As for Hirscher's recent paper, Heben claims that it is flawed and points out that it was not sent out for external peer review.

Hirscher's results, says Heben, may simply have been caused by using inferior nanotubes. "Some people think nanotubes are all the same," says Heben, "but our purity is much higher." The matter might be settled if Hirscher used exactly the same tubes as Heben, but Heben is reluctant to release his nanotubes until he obtains consistent results for hydrogen storage. At the moment, the value he gets varies depending on how it is measured and when he uses the method favoured by most other researchers, he gets only up to $4 \%$ by weight. But even if Heben does resolve this issue, he says that the bad feeling between the two parties is such that he may never be willing to supply his materials to Hirscher.

\section{Commercial brakes}

Sponsorship from industry has again complicated matters. Heben is involved in a public-private partnership designed to speed the transfer of DoE-sponsored research to commercialization. Under the terms of his agreement with Honda R\&D Americas, Heben must secure intellectual property rights

\section{more practical
hydrogen storage system would bring huge financial rewards.}

before releasing full details of his work. Some researchers suspect that this has prevented Heben from disclosing all the details of his technique for making pure nanotubes.

Heben admits that the contract "breeds mistrust", but denies that he is hiding anything. He says that by securing a patent before he announced his results, he was able to be completely open about his work. As someone who worked on experiments that failed to replicate Stanley Pons' and Martin Fleischmann's 1989 claim to have achieved cold fusion, Heben knows all about controversy. "We just want to find out what works," he says, "and if we find out it is wrong, so be it."

As Heben's salary depends heavily on funding from the DoE and his corporate partners, this attitude seems commendable. Without promising results, his funding could be decreased or withdrawn.

Doubts will remain until Heben allows other labs to test his nanotubes. But with no one else close to meeting the DoE targets, some scientists are beginning to lose faith. Many European researchers seem convinced by the finality of Hirscher's work, although some Japanese and American groups remain open-minded. "I think it is too early to conclude this story," says NEC's Iijima.

Heben will give hydrogen vehicles a big boost if he can prove his critics wrong. And if he fails, at least everyone involved will have learnt a little more about the tricky business of mixing corporate money with academicstyle research.

Catherine Zandonella is a science writer in San Francisco. 1. Iijima, S. Nature 354, 56-58 (1991).

2. Chambers, A., Park, C., Baker, R. T. K. \& Rodriguez, N. M. J. Phys. Chem. B 102, 4253-4256 (1998).

3. Ahn, C. C. et al. Appl. Phys. Lett. 73, 3378-3380 (1998).

4. Chen, P., Wu X., Lin, J. \& Tan, K. L. Science 285, 91-93 (1999).

Yang, R. T. Carbon 38, 623-626 (2000).

6. Dillon, A. C. et al. Adv. Mater. 11, 1354-1358 (1999).

. Hirscher, M. et al. Appl. Phys. A 72, 129-132 (2001). 